\title{
La educación a distancia en la profesionalización de las personas con discapacidad y privados de libertad
}

\author{
Distance Education in the Professionalization of People with \\ Disability and Prison Inmates
}

\author{
Ileana Ulate Soto ${ }^{1}$ \\ Cátedra de Investigación \\ Escuela de Ciencias de la Administración \\ Universidad Estatal a Distancia \\ San José, Costa Rica \\ iulate@uned.ac.cr \\ Elizarda Vargas Morúa \\ Cátedra de Investigación \\ Escuela de Ciencias de la Administración \\ Universidad Estatal a Distancia \\ San José, Costa Rica \\ evargasm@uned.ac.cr
}

Recibido 21 de junio de 2012 • Corregido 09 de octubre de 2012 • Aceptado 07 de noviembre de 2012

Resumen. El presente trabajo ilustra, mediante dos ejemplos, el impacto que ha tenido la Universidad Estatal a Distancia (UNED), en la atención y profesionalización de grupos vulnerables de la sociedad costarricense, tales como los privados de libertad, personas con discapacidad física, individuos de zonas muy alejadas del centro capitalino, entre otros. Se trata de dar respuesta a las preguntas: ¿Cómo ha sido la atención en esos sectores? ¿Cuáles son los retos que enfrentan las universidades a distancia para ser pertinentes? ¿De qué manera ha logrado alcanzarlos? ¿Qué impacto sobre la sociedad costarricense ejerce la UNED en la profesionalización de su población? Para el abordaje de la temática se recurre a documentación y estadísticas existentes, y se ejemplifica mediante el testimonio de dos estudiantes egresados de la UNED, elegidos con el propósito de ilustrar algunos de los diferentes casos que atiende dicha Universidad.

Palabras claves. Educación a distancia, estudiantes privados de libertad, estudiantes con discapacidad, educación a grupos vulvenrales.

\footnotetext{
Licenciada en Docencia. Máster en Administración de Negocios de la Universidad Latinoamericana en Ciencia y Tecnología. Egresada del Doctorado de la Universidad Estatal a Distancia (UNED). Encargada de Cátedra de Investigación de la Escuela de Ciencias de la Administración de la UNED, Costa Rica.

2 Licenciada en Derecho de la Universidad de Costa Rica. Máster en Administración de Negocios de la Universidad Latinoamericana en Ciencia y Tecnología. Tutora en la Cátedra de Investigación de la Escuela de Ciencias de la Administración de la Universidad Estatal a Distancia, Costa Rica.
} 
Abstract. This paper illustrates the impact of the Universidad Estatal a Distancia ${ }^{3}$ (UNED, by its Spanish acronym) in supporting and professionalizing the vulnerable groups of the Costa Rican society, including prison inmates, persons with physical disability, individuals from remote areas, among others. This study attempts to answer this type of questions: What kind of support has been provided to these sectors? What are the challenges faced by distance education universities to succeed? How does UNED have solved these challenges? What is the impact of the Universidad Estatal a Distancia on the Costa Rican society for the professionalization of its population? Documentation and statistics have been used in the study; in addition, two people who graduated from UNED were selected to illustrate the type of cases the University serves.

Keywords. Distance Education, students in detention, students with disabilities, education in vulnerable groups.

Esta investigación constata cómo la Universidad Estatal a Distancia (UNED) de Costa Rica ha podido alcanzar y profesionalizar diversos sectores sociales, a pesar de las condiciones adversas de estos: situaciones geográficas, discapacidades físicas, estados marginales especiales, enfermedades graves y privación de libertad.

Para guiar este trabajo, se han planteado las siguientes preguntas: ¿Cómo la educación a distancia contribuye en la inserción profesional universitaria de personas con diferentes tipos de limitaciones y discapacidades? ¿De qué manera la UNED está cumpliendo con los fines establecidos desde su constitución? ¿Qué aspectos deben priorizarse para alcanzar su cometido?

Para establecer el marco teórico, se define la educación a distancia, el perfil del estudiante en esta modalidad, el estudiante con discapacidad física y el privado de libertad. Además, se incluyen algunas resoluciones de la Sala Constitucional de Costa Rica, relativas a estudiantes con necesidades educativas. Posteriormente se abordan los casos de dos estudiantes egresados de la UNED. Finalmente se plantean las conclusiones y recomendaciones pertinentes.

\section{Referentes conceptuales}

\section{La educación a distancia en Costa Rica}

La educación a distancia es un modelo educativo que no exige la presencialidad, es decir, el aprendizaje se logra a pesar que el estudiante y profesor están separados por el espacio y por el tiempo. Para ello, se recurre a herramientas de comunicación -sean estas sincrónicas o asincrónicas- a fin de lograr la adecuada mediación pedagógica. Dentro de los medios utilizados se pueden mencionar: el libro de texto con actividades auto evaluativas, conocido también como unidad didáctica principal, y la plataforma virtual como medio y recurso para la realización de actividades formativas. Otros instrumentos utilizados son: los videos, las teleconferencias, material multimedia, televisión y afines.

Translator's note: It refers to the "Costa Rican Distance Education State University". 
En la UNED, las asignaturas se imparten con tres modalidades básicas.

- La primera opción se trata de cursos totalmente en línea.

- La segunda opción ofrece, por una parte, los llamados cursos híbridos en línea, y por otra, sesiones previamente establecidas entre el tutor y los estudiantes.

- Por último, se ofrecen cursos regulares en los cuales el estudiante realiza, de manera independiente, las lecturas y actividades que se le encomiendan y, con el fin de aclarar dudas, tiene la posibilidad de una reunión con el profesor o tutor una vez por mes. Esto es lo que se conoce como tutorías presenciales. No son obligatorias para ningún estudiante, ya que se dan a fin de aclarar dudas que hayan surgido del estudio individual de los contenidos de cada asignatura.

La oferta académica bajo los términos antes descritos constituye un elemento muy importante dentro del éxito del modelo a distancia, ya que favorece el aprendizaje individualizado, de esta forma cada educando es auto dirigido y auto controlado.

En parte, el éxito del sistema a distancia se puede resumir en dos términos "flexibilidad"y "autogestión", tal y como se señala en el siguiente texto:

La flexibilidad en cuanto al manejo de sus propios tiempos por parte del estudiante, la ausencia del requisito de asistencia periódica a clase, la posibilidad de seguir los estudios desde cualquier parte a donde el alumno se vea obligado a trasladarse por distintas razones laborales o personales, en definitiva el alto grado de autonomía de que el educando goza en el sistema, constituye a la educación a distancia en una opción apropiada para los tiempos que corren, ya que permite compatibilizar las exigencias de capacitación con las limitaciones espaciotemporales que impone la vida contemporánea. (Solari y Monge, 2004, p. 4)

Otras bondades de los modelos de educación a distancia son:

- Permitir al estudiante avanzar a su propio ritmo de aprendizaje

- Utilizar diferentes materiales, auditivos, visuales, audiovisuales

- Estudiar en forma individual

- Ejercitarse en el lugar y tiempo disponible por el alumno

- Ser flexible

- Ahorrar costos y desplazamientos. (Cabero, 2006)

Se puede decir, entonces, que las características de estos modelos educativos favorecen la inclusión de estudiantes con necesidades especiales en los sistemas universitarios, ya que les permite auto gestionar su aprendizaje, mediante parámetros establecidos previamente. 
Otras oportunidades que la educación a distancia plantea son las de promover la accesibilidad de la educación a la comunidad, proveer un sistema educativo más personalizado, es decir, que responda a las necesidades formativas de cada individuo. Es un sistema flexible que se refleja y adapta a las necesidades diversas. Tiene como fin desarrollar sistemas tecnológicos para elaborar materiales y recursos con altos niveles de interactividad (Sangrà, 2002).

Con el fin de sintetizar de manera gráfica el modelo de educación a distancia, se detallan, en la figura 1, las principales fortalezas, oportunidades, debilidades y amenazas del sistema de educación a distancia en Costa Rica, con base en los autores ya mencionados.

\begin{tabular}{|c|c|c|c|c|}
\hline \multicolumn{2}{|c|}{ FORTALEZAS } \\
\hline $\begin{array}{c}\text { Liberación al docente } \\
\text { de labores de custodia } \\
\text { y favorecimiento a la } \\
\text { realización de tareas } \\
\text { más significativas }\end{array}$ & $\begin{array}{c}\text { Autodirección y } \\
\text { autocontrol de } \\
\text { educandos de su } \\
\text { aprendizaje }\end{array}$ & $\begin{array}{c}\text { Divulgación masiva } \\
\text { de la información por } \\
\text { diversos medios }\end{array}$ & $\begin{array}{c}\text { Ahorro de costos de } \\
\text { desplazamiento }\end{array}$ & Flexibilidad \\
\hline
\end{tabular}

\begin{tabular}{|c|c|c|c|c|}
\hline \multicolumn{5}{|c|}{ OPORTUNIDADES } \\
\hline $\begin{array}{l}\text { Mantener las carreras } \\
\text { acreditadas ante el } \\
\text { SINAES en el caso de } \\
\text { Costa Rica }\end{array}$ & Avance tecnológico & $\begin{array}{l}\text { Crecimiento acceso a } \\
\text { los diversos medios de } \\
\text { comunicación en el país }\end{array}$ & $\begin{array}{l}\text { Sistema que disminuye } \\
\text { la discriminación racial, } \\
\text { social y física }\end{array}$ & $\begin{array}{c}\text { Accesibilidad para la } \\
\text { educación superior de } \\
\text { adultos }\end{array}$ \\
\hline
\end{tabular}
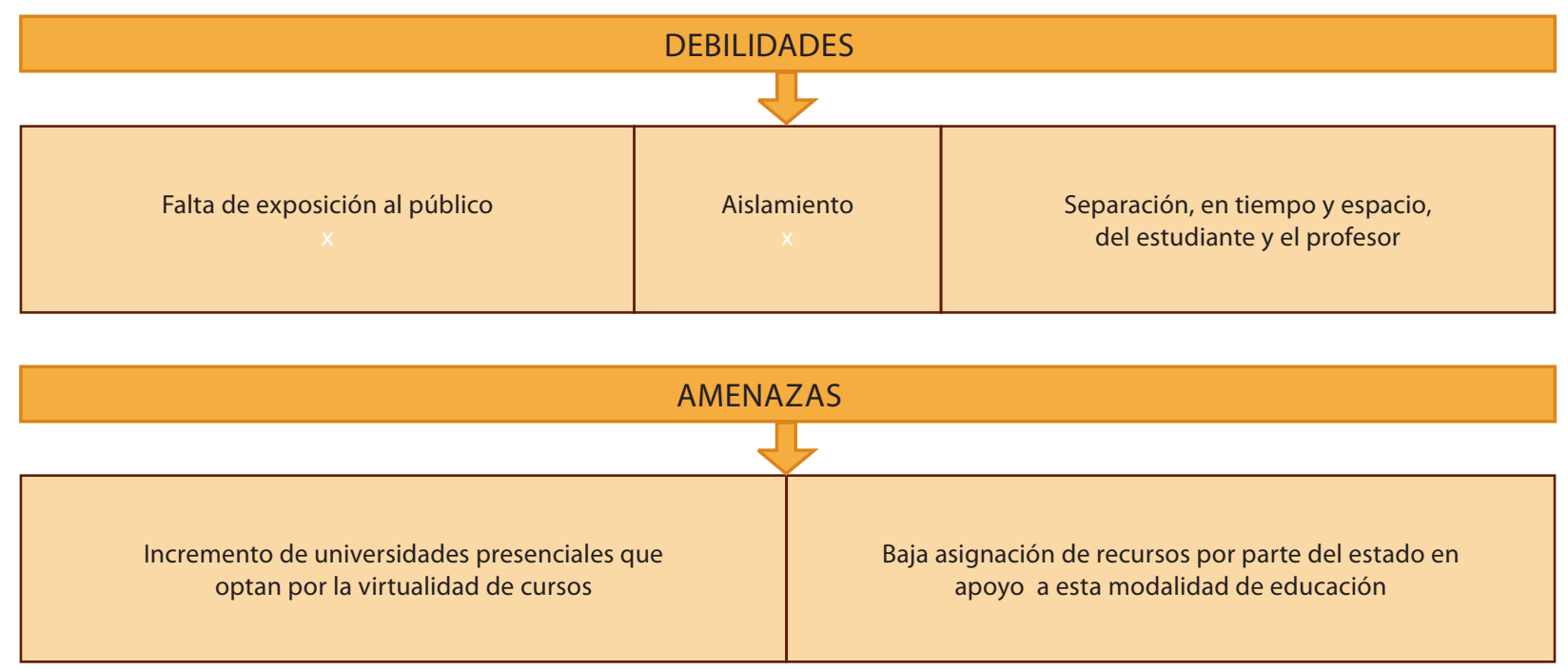

Figura 1. Fortalezas, oportunidades, debilidades y amenazas de la educación a distancia. Elaboración propia con base en los autores mencionados. 
Lejos de ser un sistema educativo subsidiario y emergente, gracias a la tecnología de la información; a la comunicación; al cambio en los hábitos de los individuos; a la concepción de la educación como un proceso que se extiende a lo largo de la vida, aún en situaciones menos favorecedoras, la educación a distancia es la educación que ha venido posicionándose, como una real, oportuna y efectiva forma de educación. Tanto es así que las universidades tradicionales están incorporando la tecnología de la información y la comunicación en su oferta educativa y en sus métodos docentes, investigativos y de gestión (Sangrà, 2002).

Es necesario señalar además que, para ofrecer carreras que estén acordes con el mundo competitivo que nos rodea, la flexibilidad que caracteriza a estos sistemas de educación superior tiene que ir de la mano con la calidad, la cual no puede verse amenazada. Tomando en cuenta esta consideración, la UNED se ha sometido a los procesos de acreditación del Sistema Nacional de Educación Superior (SINAES), ente que oficialmente acredita la existencia de un nivel apropiado para las carreras ofrecidas tanto a nivel privado como público. Las carreras acreditadas al 2012 en la UNED son: profesorado y bachillerato en Enseñanza de las Ciencias Naturales y en la Enseñanza de la Matemática, bachillerato y licenciatura en Informática Educativa y en Manejo de Recursos Naturales y seis de los énfasis de la Escuela de Ciencias de la Administración (contabilidad, finanzas, mercadeo, recursos humanos, producción y dirección de empresas) (Ramírez, 2012).

\section{La Universidad Estatal a Distancia}

La Universidad Estatal a Distancia (UNED) es la universidad pionera de la educación a distancia en Costa Rica. Nace en 1977, mediante la ley No. 6044 (UNED, 1977), como una nueva opción de estudio para la población costarricense.

Su ley constitutiva, en el artículo 2 señala, como un objetivo de esta Universidad, brindar acceso a la educación superior, mediante métodos idóneos y flexibles, a quienes no hubieren podido incorporarse al sistema formal universitario.

Por su parte, la universidad, en su Estatuto Orgánico del 30 de mayo del 2000, publicada en el diario oficial La Gaceta No. 201, del viernes 20 de octubre del 2000 (UNED, 2009) establece, en su artículo segundo, que la UNED tiene como objetivo proporcionar educación superior, principalmente mediante la utilización de técnicas de educación a distancia y atender, con preferencia, a aquellos sectores de la población que, por razones geográficas, de trabajo o de otro tipo, no puedan asistir a los otros centros de educación superior.

Desde su creación ha presentado un crecimiento constante, reflejado en el hecho de que, para el 2012, cuenta con treinta y cuatro centros universitarios distribuidos por todo el país, incluso en lugares alejados del área metropolitana, entre ellos: Talamanca, Pavón, Puerto Jiménez, Upala, San Vito, Osa, Jicaral y otros. 
URL: http://www.una.ac.cr/educare

Actualmente, como se puede observar en la figura 2, la UNED es la segunda institución pública con mayor matrícula del país:

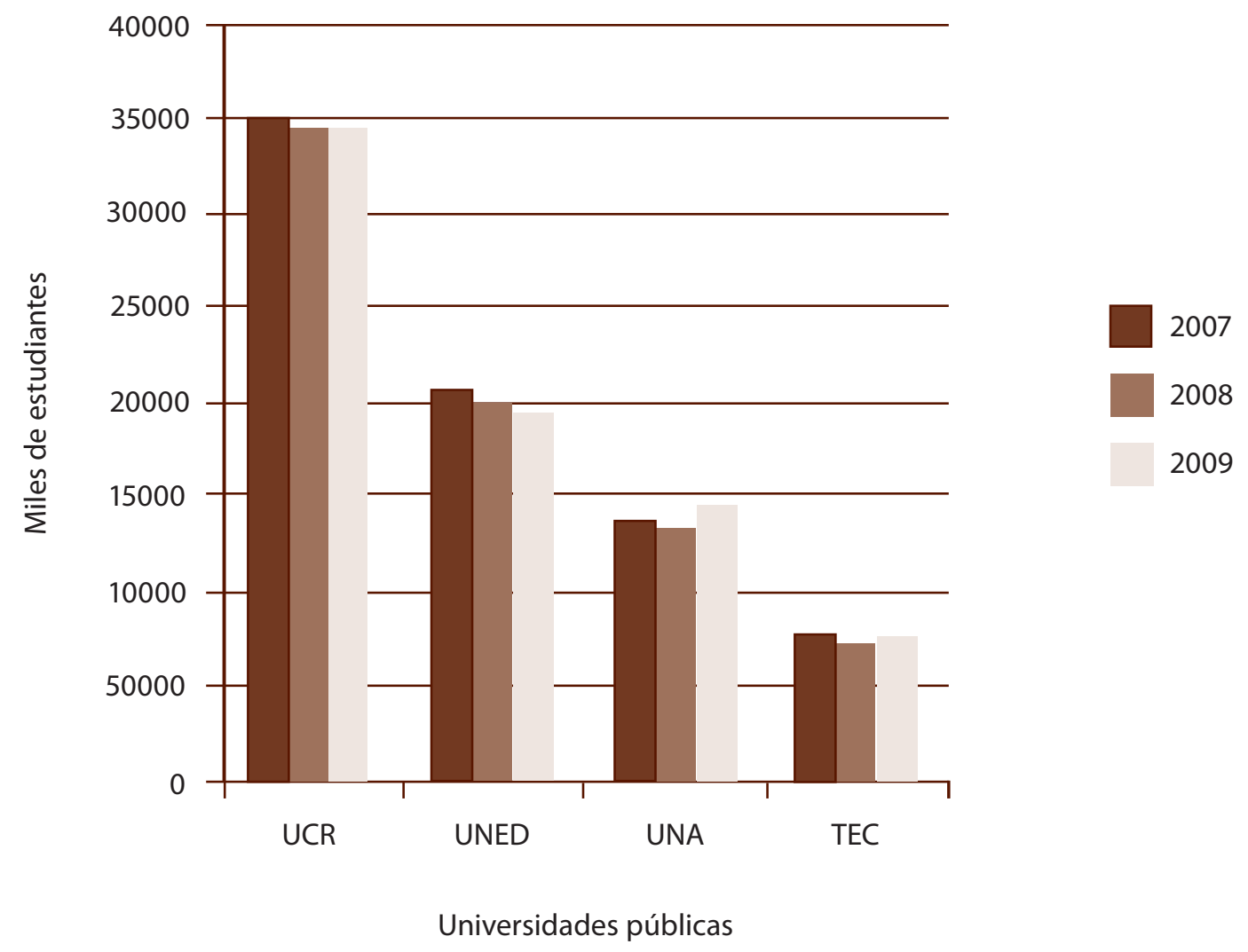

Figura 2. Matrícula del I ciclo lectivo de universidades públicas costarricenses del 2011.

Elaboración propia a partir del informe Estado de la Nación en Desarrollo Humano Sostenible (2011). 
En cuanto a los títulos entregados por las universidades, la UNED ocupa el tercer puesto. En otras palabras, la UNED es la universidad pública que ocupa el puesto número tres en graduados de las carreras que oferta.

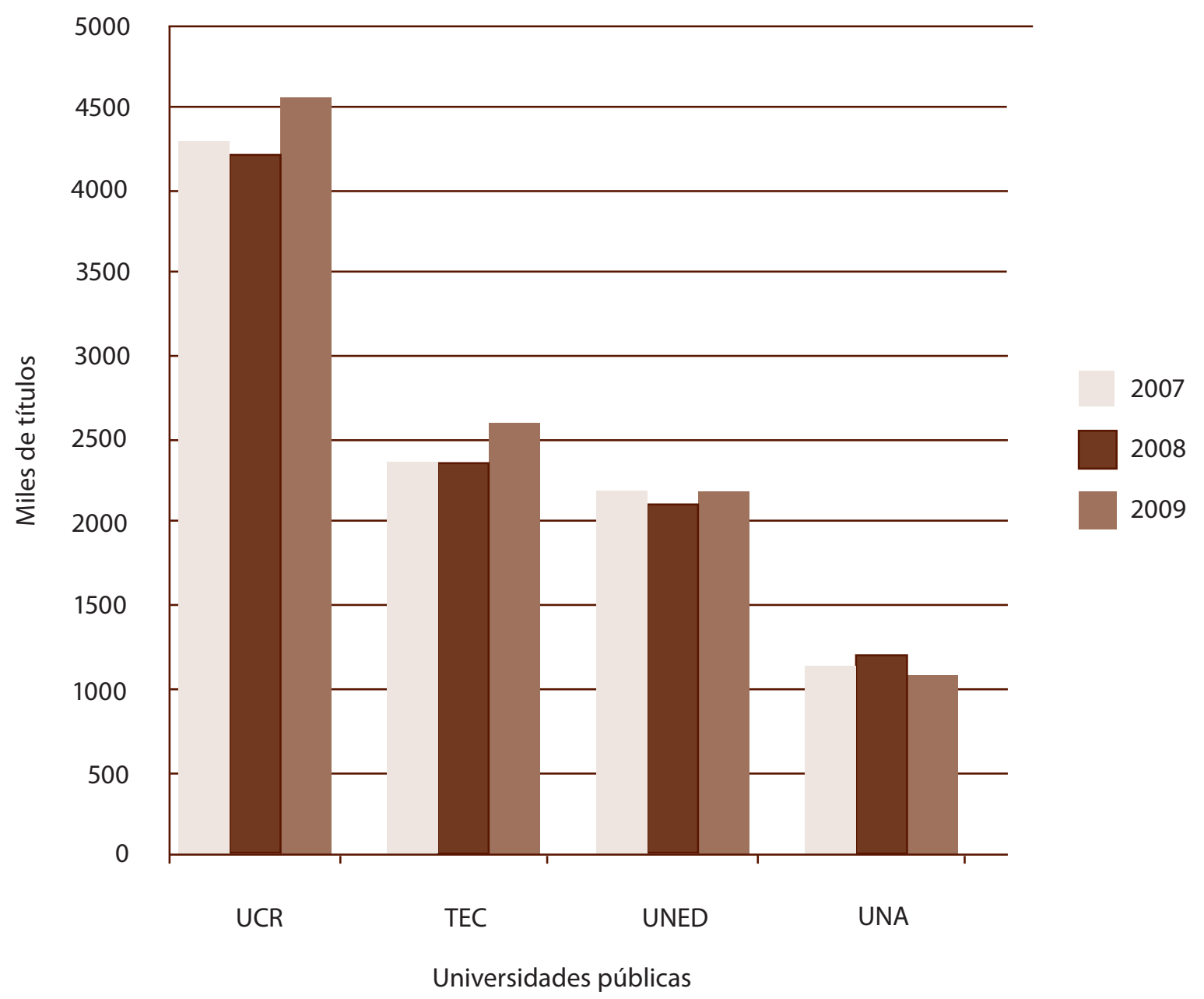

Figura 3. Títulos entregados por las universidades públicas costarricenses

Elaboración propia a partir del informe del Estado de la Nación en Desarrollo Humano Sostenible (2011).

Los indicadores anteriores evidencian la importante participación de la UNED en la formación de profesionales. 


\section{Perfil del estudiante a distancia}

\section{Generalidades}

De acuerdo con García (1999), el proceso de educación a distancia debe estructurarse teniendo como eje central al estudiante; esto implica considerar las motivaciones, los estilos de aprendizaje y los aspectos psicológicos de cada individuo.

Agrega el autor que, por lo general, los sistemas a distancia dirigen sus esfuerzos para ofrecer formación a personas adultas, quienes son responsables de su propio aprendizaje. La educación de adultos se basa en las experiencias previas, la capacidad, los hábitos, las metas, las actitudes, y las estrategias de autorregulación y autocontrol de su proceso. Por estas razones, las metodologías empleadas en el proceso de enseñanza y aprendizaje de esta población difieren significativamente de las utilizadas en la educación presencial. Una mediación pedagógica eficaz debe lograr que el proceso, que se realiza de manera autónoma e independiente, se ajuste a cada necesidad; a los intereses individuales; al tiempo, motivaciones y estilos de aprendizaje de cada participante; de tal manera que el currículo tiene que ser flexible.

En la siguiente figura se esquematizan algunas de las características del estudiante a distancia que logra culminar su proceso de aprendizaje mediante este método.

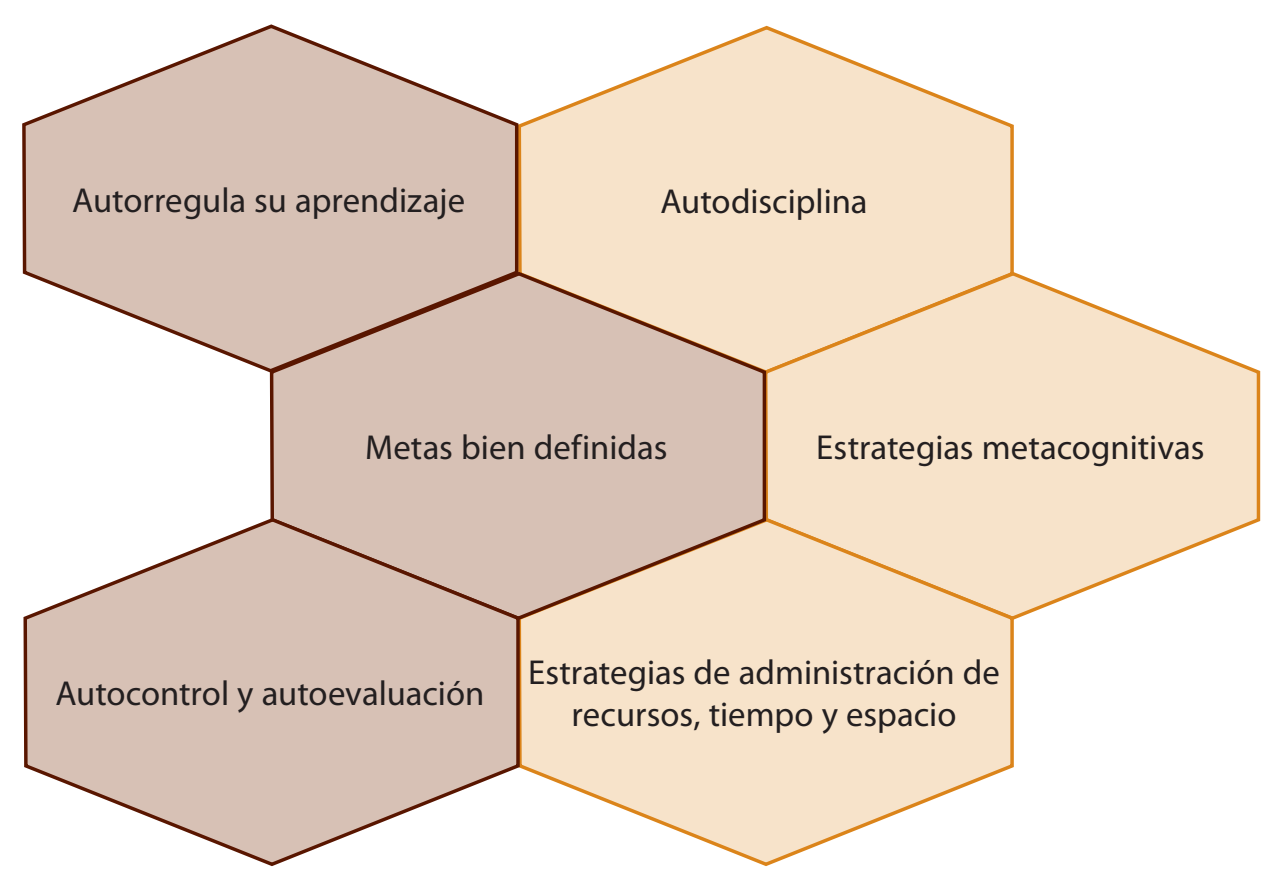

Figura 4. Características del estudiante a distancia. Elaboración propia a partir de García (1999). 
Es importante destacar que, pese a que los sistemas a distancia están dirigidos a personas adultas, también existen otras poblaciones beneficiadas como: los privados de libertad, los que residen en zonas muy alejadas y que por dificultades financieras no podrían ingresar a la educación superior, personas de la tercera edad, aquellos que tienen necesidades especiales de aprendizaje o con alguna limitación física, hablantes no nativos del español, entre otras. Poblaciones todas con necesidades específicas que pueden ser atendidas por la formación a distancia, que ofrece, así, un medio para el acceso a la educación superior.

A continuación se presentarán datos que detallan las características del estudiantado de la UNED con necesidades educativas especiales, tal es el caso de privados y privadas de libertad y estudiantes con discapacidades físicas.

\section{Perfil del estudiante UNED}

Parte de la población estudiantil que conforma la UNED, la integran los privados de libertad. Seguidamente se mostrarán datos que reflejan la matrícula por centro penitenciario y por año.

Tabla 1

Número de estudiantes matriculados en la Universidad Estatal a Distancia según CAl por cuatrimestre en el año 2011 (Los estudiantes son privados de libertad)

\begin{tabular}{lcccc}
\hline CAI & $2011-I$ & $2011-I I$ & $2011-I I I$ & 2011 \\
\hline Calle Real & 19 & 8 & 5 & 32 \\
Cocorí & 15 & 14 & 14 & 43 \\
El Buen Pastor & 19 & 15 & 10 & 44 \\
El Roble & 8 & 8 & 8 & 24 \\
La Reforma/El Virilwla & 66 & 83 & 60 & 209 \\
La Leticia & 12 & 10 & 9 & 31 \\
La Marina & 9 & 12 & 10 & 31 \\
San Agustín & 4 & 6 & 5 & 15 \\
San Isidro & 8 & 8 & 8 & 24 \\
San Rafael & 11 & 10 & 11 & 32 \\
San Sebastián & 0 & 1 & 1 & 2 \\
Sandoval & 11 & 10 & 7 & 28 \\
San Ramón & 1 & 1 & 1 & 3 \\
\hline TOTAL & 183 & 186 & 149 & 518 \\
\hline
\end{tabular}

CAl: Centro de atención institucional. Información suministrada por J. P. Camacho (comunicación personal, Dirección de Asuntos Estudiantiles de la UNED, 10 de abril, 2012). 
URL: http://www.una.ac.cr/educare

Se observa, a través de los datos anteriores, que durante el año 2011, 518 privados de libertad se matricularon en alguna de las carreras que oferta la universidad.

No caben dudas de los beneficios que le trae, a una persona privada de libertad, el ocupar su tiempo en actividades como el estudio. De conformidad con Scarfó (1997), la educación en la cárcel persigue tres objetivos inmediatos:

1. Mantener a los presos ocupados provechosamente.

2. Mejorar la calidad de vida en la cárcel.

3. Conseguir un resultado útil, oficio, conocimiento, comprensión, actitudes sociales y comportamientos.

Como ciudadanos, es importante reflexionar sobre el hecho de que casi todas las personas que han delinquido y se encuentran prisioneras serán puestas en libertad en la misma sociedad en la que han delinquido. De tal manera que, cuantas mejores oportunidades de reintegración con éxito se les otorgué, mayor seguridad para la sociedad de que no reincidirán en su accionar negativo. En este sentido, la educación contribuye al proceso de integración social, pues el proceso formativo es susceptible de producir cambios en las actitudes (Scarfó, 1997).

Aunado a los beneficios ya citados y de conformidad con la normativa costarricense, los condenados a pena de prisión pueden solicitar, una vez cumplida la mitad de la pena impuesta en sentencia ejecutoriada (Código Penal, sección III, de la libertad condicional, requisitos, artículo 65, s. f.), la libertad condicional. Para ello, deben cumplirse algunos requisitos como el hecho de que el condenado pruebe su buena conducta y adquisición de algún oficio, que le permitan una vida regular (artículo 65). Esto constituye un aliciente más para el privado de libertad.

El estudio en la cárcel tiene sus particularidades. Dos de las dificultades que debe enfrentar un privado de libertad que desee estudiar son la falta de tutorías presenciales y la falta de espacio privado para estudiar (Castrillo, 2012). Estas dos limitaciones se comprobaron con la entrevista que consta más adelante brindada por el privado de libertad.

\section{Estudiantes con necesidades educativas especiales}

Otro sector que se atiende son los alumnos con necesidades educativas especiales. En el primer cuatrimestre del 2012 se inscribieron 251 con diversas discapacidades, como se indica en la tabla 2: 
Tabla 2

Estudiantes con necesidades educativas especiales

\begin{tabular}{lc}
\hline Discapacidad & Total de estudiantes \\
\hline Auditiva & 11 \\
Auditiva-Baja visión & 1 \\
Baja visión & 19 \\
Baja visión-Física & 1 \\
Baja Visión-Problema de aprendizaje & 1 \\
Ceguera & 14 \\
Física & 36 \\
Física-Problema de aprendizaje & 2 \\
Múltiple & 1 \\
Problema de aprendizaje & 159 \\
Problema de aprendizaje-Baja visión & 2 \\
Salud & 4 \\
\hline
\end{tabular}

Nota: Información suministrada por Y. Morales (comunicación personal, Dirección de Asuntos Estudiantiles de la UNED, 10 de abril, 2012).

Estudiantes con estas condiciones en algún momento requieren adecuación curricular, la cual se define como:

(...) el conjunto de ajustes o modificaciones que se introducen en la planificación educativa (...) para favorecer la adquisición de las capacidades básicas contempladas en la propuesta curricular oficial, a la vez que permite ofrecer una respuesta educativa integral a las necesidades educativas de los estudiantes de la diversidad. (Arnaiz, 2000, p. 151)

Las políticas educativas universitarias deben redactarse de manera tal que permitan, en igualdad de oportunidades, el acceso a la educación superior a los sectores discapacitados, siempre y cuando posean las condiciones intelectuales que les permitan mantenerse y proseguir estudios de educación universitaria (esta posición es congruente con la posición de la Sala Constitucional, como se indicará más adelante). 
Partiendo de la Convención sobre los Derechos de las Personas con Discapacidad (s. f.) "(...) discapacidad es un concepto que evoluciona y que resulta de la interacción entre las personas con deficiencias y las barreras debidas a la actitud y al entorno que evitan su participación plena y efectiva en la sociedad, en igualdad de condiciones con las demás" (párr. 5). Este enfoque enfatiza no la limitación de la persona, sino las barreras y obstáculos que existen en el entorno para que la persona con discapacidad participe plenamente en todos los ámbitos de la vida.

Históricamente, las personas con discapacidad han tenido menor acceso a la educación, a causa de las diversas barreras sociales, estereotipos y al predominio de modelos teóricos y paradigmas que no aceptaban a las personas con discapacidad como sujetos de derechos que forman parte de la sociedad.

Según información del Registro Estadístico en Discapacidad, se estima que aproximadamente un $60 \%$ de las personas con discapacidad con 18 años o menos tiene la primaria completa o se encuentra en ella; $24 \%$ de edades entre 19 y 25 años han logrado terminar la secundaria y solo el 14\% lograron ingresar a la educación superior.

De los que ingresan a centros educativos; el 90.1\% lo hacen a una institución pública, el 9.9\% restante a un sistema educativo privado.

\section{Enfoque legal}

Desde el punto de vista legal, es importante mencionar que los países latinoamericanos han realizado ajustes a las normativas pertinentes al tema con el fin de disminuir la discriminación de las personas con alguna discapacidad a los sistemas educativos. Como parte de estos, cabe mencionar la Convención sobre los derechos de las personas con discapacidad, realizada por las Naciones Unidas, celebrada el 13 de diciembre del 2006, con la participación de casi todos los países de la región Latinoamérica y Cuba, salvo Venezuela (Asamblea General de las Naciones Unidas, 2006). Este convenio se ha convertido en un valioso instrumento que señala enfáticamente el derecho de los discapacitados para accesar a una educación sin discriminación y sobre la base de igualdad de oportunidades para todos.

El compromiso de los Estados participantes es asegurar un sistema educativo inclusivo en todos los niveles y a lo largo de toda la vida. En este se hace necesario proyectar:

- El potencial humano, respetando los derechos humanos, las libertades fundamentales y la diversidad, así mismo se debe dignificar al ser humano y su autoestima.

- El desarrollo de la personalidad, los talentos y la creatividad, así como sus aptitudes mentales y físicas.

- La integración libre de las personas con discapacidad a la sociedad. 
Si las naciones se comprometen a favorecer esta integración, se asegura no excluir a ninguna persona por motivos de su condición; así mismo se propicia que esta población acceda a una educación inclusiva de calidad, gratuita y en igualdad con respecto a su entorno; por tanto, para fomentar el máximo desarrollo académico y social, se requiere que se realicen los ajustes necesarios, en virtud de las necesidades individuales, y se preste el apoyo preciso en el marco del sistema general de educación.

Los Estados partes asegurarán que los individuos tengan acceso general a la educación superior, la formación profesional, la educación para adultos y el aprendizaje durante toda la vida, sin discriminación y en igualdad de condiciones con las demás. Para tal fin las naciones participantes aseguran que se realicen ajustes razonables para las personas con discapacidad.

\section{Caso Costa Rica}

En el caso de Costa Rica, se debe mencionar que, de acuerdo con el Informe país sobre el cumplimiento de la convención (Consejo Nacional de Rehabilitación y Educación Especial, 2008), el Estado ha planteado políticas de educación que incluyen el acceso a estudiantes con necesidades especiales. En el Plan Nacional de la Educación Superior Universitaria Estatal 2011-2015 (Consejo Nacional de Rectores, Oficina de Planificación de la Educación Superior, Comisión de Directores de Planificación, 2011) se impulsan proyectos tendientes a favorecer las condiciones de las personas con discapacidad en las universidades, con el fin de lograr una mayor cobertura y equidad. Otra de las acciones que se han ejecutado es la creación de la Comisión Interuniversitaria de la Accesibilidad a la Educación Superior, cuya finalidad es la articulación de las políticas de acceso a la educación superior que intervienen en el proceso de admisión y permanencia en los centros de estudio, y propician el acceso en igualdad de oportunidades.

Un modelo de universidad accesible para todos requiere una legislación que incluya las necesidades de todos sus miembros; por tanto, estas instituciones tienen el compromiso público de promover y divulgar el cumplimiento de cualquier reglamento o normativa interna, nacional e internacional que pretenda crear condiciones de igualdad de oportunidades para todos.

Es claro que los retos pendientes para lograr la inclusión educativa son muchos. Los datos que a partir de ahora se obtengan con el Registro Estadístico en Discapacidad deberán servir para que las personas que toman decisiones incorporen políticas y se desarrollen acciones para que ningún costarricense con discapacidad quede excluido del sistema general de educación. El país debe avanzar por el camino que señala la Convención Internacional de Derechos de las Personas con Discapacidad, Ley 8661, que en su artículo 24 indica "(...) las personas con discapacidad puedan acceder a una educación primaria y secundaria inclusiva, de calidad y gratuita, en igualdad de condiciones con las demás, en la comunidad en que vivan" (Organización de las Naciones Unidas [ONU] s. f., p. 19). 
URL: http://www.una.ac.cr/educare

\section{Resoluciones de la sala constitucional que impactan las situaciones de estudiantes con adecuaciones curriculares en las universidades}

Las resoluciones de la Sala Constitucional de interés para el presente artículo se refieren a los derechos y obligaciones de las instituciones para la atención de estudiantes con necesidades especiales en recintos universitarios.

Al mes de abril del 2012, se rescatan resoluciones que indican que, si bien las universidades pueden aceptar estudiantes con necesidades especiales, no existe una obligación a nivel universitario de realizar adecuaciones curriculares significativas, como tampoco hay obligación de recibir estudiantes que por su situación mental puedan poner en riesgo el resto de los alumnos.

A continuación los siguientes extractos de resoluciones:

La universidad no está obligada a recibir estudiantes con adecuaciones curriculares significativas

Un estudiante con adecuación curricular significativa, interpuso un recurso ante la Sala Constitucional. Aducía tener problemas de aprendizaje, lo cual hizo que durante sus estudios de primaria y secundaria le aplicaran adecuaciones curriculares. Posterior a ello, intentó matricularse en la UNED para llevar el ciclo básico de Generales, pero la Directora le denegó su gestión, con el argumento de que la institución no aceptaba estudiantes que requirieran adecuación curricular significativa. Mediante el voto 10727-10 (Sala Constitucional de la Corte Suprema de Justicia de la República de Costa Rica, 2010) la sala resolvió sin lugar el recurso interpuesto por el estudiante, en el mismo sentido que otra sentencia anterior número 1162108, ya que la universidad no está obligada a recibir estudiantes con adecuaciones curriculares significativas (Sala Constitucional de la Corte Suprema de Justicia de la República de Costa Rica, 2008b).

El estudiante universitario debe tener aptitudes intelectuales que hacen incompatible la implementación de adecuaciones curriculares significativas

El caso en estudio trató de un estudiante con retardo mental que solicitó aplicar adecuaciones curriculares significativas, tutor en todas las materias y adecuación en los exámenes, conforme a la Ley No. 7600.

Mediante el voto 11621-08, la Sala indicó que lo pretendido por el recurrente era improcedente, pues los estudios universitarios responden a otra filosofía y otros fines, en los cuales no es posible la aplicación de la adecuación curricular significativa, por versar específicamente sobre su capacidad intelectual para asimilar el contenido de las materias que conforman la carrera. 
De conformidad con dicho voto, la educación superior universitaria va encaminada a formar profesionales, quienes deben tener un nivel académico adecuado para el ejercicio de la profesión de la que se trate. Es indispensable que el estudiante posea, entre otras condiciones, las aptitudes intelectuales necesarias para su desempeño, situación que hace incompatible la implementación de adecuaciones curriculares significativas.

La atención de estudiantes con necesidades especiales no puede ser en perjuicio de la seguridad personal del resto de los estudiantes

El recurso ante la Sala fue planteado por un estudiante con problemas psicológicos y con tratamientos médicos que le ocasionan una adecuación curricular. Alegó que la universidad le negó el ingreso por lo que interpuso el recurso. Al respecto, la sala mediante el voto 10944-08 (Sala Constitucional de la Corte Suprema de Justicia de la República de Costa Rica, 2008a) consideró que las medidas tomadas por la universidad habían sido razonables para garantizar la seguridad personal de los estudiantes, dado que en varias ocasiones, según quedó constando, el estudiante había mostrado una conducta impropia contra estos.

La Universidad debe adoptar las medidas necesarias y acciones con el fin de facilitarles a sus estudiantes el disfrute pleno de las adecuaciones curriculares requeridas

Este caso fue presentado ante la Sala por una estudiante no oyente. La misma necesita usar audífonos como ayuda técnica y se comunica por medio de lenguaje de señas (Lesco). Estudia Educación Especial en la Universidad recurrida, la cual le otorgó una beca del 100 \%, condicionada a que realice un trabajo de 40 horas por cuatrimestre. Sin embargo, la estudiante alegó que no había podido cumplir con la exigencia y solo había podido laborar 19 horas, porque no había un trabajo apto para ella, lo cual era responsabilidad de la Universidad. Manifestó que a una semana de concluir el cuatrimestre, no había podido terminar, pese a que había solicitado la adecuación desde el $2^{\circ}$ cuatrimestre. Agrega que en la carrera no hablan en Lesco, no imparten el curso y los profesores hablan muy rápido, sin considerar su condición de minusválida, lo cual le dificulta leerles los labios, además al presentar películas no se incorporan subtítulos.

Este recurso fue declarado con lugar, mediante el voto 2773-09 (Sala Constitucional de la Corte Suprema de Justicia de la República de Costa Rica, 2009), en consecuencia, se ordenó al Presidente de la Asociación de la Universidad adoptar las medidas necesarias y ejecutar las acciones pertinentes, a fin de facilitarle a la amparada el disfrute pleno de las adecuaciones curriculares que requiere en razón de su discapacidad. 
URL: http://www.una.ac.cr/educare

\section{Metodología}

La metodología utilizada para la presente investigación es cualitativa, con un enfoque descriptivo. Para la misma se recurrió a las siguientes técnicas:

- Análisis documental

- Revisión y análisis de la información brindada por autoridades universitarias y documentos institucionales

- Información suministrada por los funcionarios Yolanda Morales Quesada y Juan Pablo Camacho, de la Dirección de Asuntos Estudiantiles de la UNED

- Entrevista a los estudiantes

- Revisión de indicadores del Estado de la Nación, Ministerio de Educación, la UNED y el Registro Estadístico en Discapacidad

- Sistematización de experiencias

- Valoración del análisis y las experiencias

- Esta investigación se ilustra con dos casos

\section{Desarrollo de los estudios de caso}

A continuación, el presente estudio se ilustra con el resumen de las experiencias, en la UNED, de dos estudiantes que presentaron su defensa de tesis en el año 2011, ambos en Administración de Empresas.

\section{Privado de libertad}

En el mes de agosto del año 2011, en el Centro Universitario de Puntarenas, presentó su defensa de tesis para optar por el grado académico de licenciado en Administración de Empresas con énfasis en Dirección de Empresas.

A sus 47 años, le acompañaron en este acto su mujer y su hijo por nacer, además de autoridades del Ministerio de Justicia y del propio centro universitario.

Inició sus estudios en el año 88 en el centro universitario de Ciudad Neilly. Manifiesta que, en ese entonces, el centro no brindaba las condiciones mínimas para estudiar, pues era pequeño, el calor insoportable y los materiales que utilizaba la universidad de muy bajo nivel. En esas condiciones se retiró de clases. Sin embargo, para mediados de los 90, según su opinión, la UNED realizó un giro en la forma de brindar el aprendizaje: 
"con materiales más dinámicos, de grandes editoriales extranjeras, de igual manera, la EUNED modificó sus estándares y confeccionaron libros de excelente calidad, lo que me motivó a volver a la UNED".

Nuevamente tuvo que retirarse por problemas económicos hasta el año 2001, cuando obtuvo una beca y pudo continuar, sin embargo:

“...En noviembre del año 2006, por la ambición al dinero, participe en una red de tráfico de drogas, siendo detenido por segunda vez y enviado al centro penal de Puntarenas, centro en el cual continué mis estudios".

De acuerdo con su experiencia:

"Estudiar en un centro penitenciario presenta muchas limitaciones, ya que los que trabajan (por lo general son los mismos que estudian) tienen un horario de ocho horas de labor, con una remuneración de 15,000 colones al mes. Los compañeros fuman drogas todo el día, la televisión pasa encendida 18 horas diarias".

En el CAIP de Puntarenas, a los estudiantes a partir del 2011, se les concedió la oportunidad de salir del recinto de los ámbitos y estudiar en los comedores. Se logró, así, obtener un poco de aislamiento para poder estudiar (5 horas diarias):

"Dentro del pabellón los que estudiamos conseguimos bombillos para estudiar de noche, pero los oficiales de seguridad nos los quitan y los quiebran cada vez que hacen una requisa".

Algunos de los problemas que manifiesta enfrentó en el Centro Penal son:

"1. Falta de tutor: mientras esté encarcelado, la UNED jamás enviará un tutor a la cárcel a resolver dudas de los estudiantes, por las razones que se deseen argumentar, el caso es que no habrá tutor.

2. Prohibición de acceso a Internet.

3. Falta de comunicación con los encargados de cátedra. La falta de comprensión de los encargados de cátedra, que no quieren saber nada de los privados de libertad, y por ello no reciben llamadas de los centros penales y, a su vez, son esquivos en realizar adecuaciones en las formas de evaluación.

4. Falta de adecuaciones en la forma de evaluación". 
URL: http://www.una.ac.cr/educare

Para concluir indicó:

"Considero que la UNED es una maravillosa institución que me ha brindado una gran oportunidad en la vida para mejorarme como persona y convertirme en un buen profesional. En la balanza de los pros y contra, es mucho más lo positivo, tienen grandes personas que se preocupan realmente por los estudiantes, y que la falta de apoyo de otros funcionarios, es por la falta de exponerles las necesidades y deseos que tienen los privados de libertad por estudiar y ser útiles a la sociedad".

"El estudio es la mejor manera de recuperar a una persona que cometió un delito, ya que su superación la observan otros compañeros y ven que sí es posible salir adelante".

\section{Estudiante de zonas alejada del centro urbano y con problemas de salud}

El caso es de una estudiante de San Vito de Coto Brus (Puntarenas). Su testimonio es el siguiente:

El 14 de marzo del 2011 me diagnosticaron cáncer de mama, este resultado implica enfrentar un tratamiento bastante fuerte, que en el caso de otras universidades presenciales, hubiese implicado un retraso en el avance profesional que estaba a punto de conseguir, no obstante, el sistema de educación a distancia me permitió alcanzar las metas antes propuestas, pese a la situación de salud que enfrenté. El sentimiento hacia los funcionarios de la UNED ha sido siempre de entero agradecimiento, ya que fueron muy comprensivos con mi situación y me brindaron su ayuda en todo momento. Para ejemplificar puedo citar el hecho que el día asignado para la defensa de tesis coincidió con la día en que me iban a realizar la operación, muy amablemente en la Universidad me ayudaron y me suspendieron la fecha, hasta el momento en que ya hubiera pasado lo más duro del tratamiento.

Soy de una zona rural de Costa Rica, por tanto los traslados constantes a las sedes o centros universitarios, implican en mi caso una inversión de tiempo de casi dos horas y media; a esto se debe agregar la agravante de que los sistemas de transporte público no son constantes y con horarios limitados. Por tanto, el uso de los medios de comunicación electrónica como recurso para el avance y revisión de los anticipos de mi proyecto de investigación fueron fundamentales, la realimentación por parte de tutor asignado haciendo uso de las herramientas tecnológicas que ofrece la universidad y todos los otros recursos de mediación pedagógicas que ofrece la cátedra de investigación favorecieron el buen desempeño de mi trabajo final de graduación. 
Estando aún en proceso de recuperación, recibí la llamada por parte de los funcionarios de la Universidad para determinar si era oportuno fijar fecha para defensa de la tesis - ya que anteriormente se había suspendido el proceso por la misma razón-. La defensa era el último requisito para obtener el grado de Licenciada en Administración con énfasis en Banca y Finanzas, por ese motivo respondí que si; sin embargo, el traslado a la capital para realizar este evento era muy difícil, debido a mi situación de salud. Por ese motivo, todos los miembros del Tribunal se trasladaron hasta el Centro Universitario de Ciudad Nelly, en donde realicé la defensa y presentación de mi trabajo final de graduación y logré culminar exitosamente mi plan de estudios. Este detalle demostró la solidaridad y calidad humana de los funcionarios que integran la Universidad Estatal a Distancia.

Realmente me siento muy agradecida, satisfecha y orgullosa de haberme podido graduar de tan prestigiosa institución como lo es la UNED.

El sistema de educación a distancia hace que el estudiante desarrolle habilidades de autocontrol, autodisciplina y autodirección, valores que han influido en el desarrollo de mi vida profesional, al punto que en estos momentos me encuentro desempeñándome al $100 \%$ en mi carrera, con todos los reconocimientos posibles en cuanto a salario, puestos, funciones, parte de haber podido obtener mi actual empleo fue que la empresa para la cual laboro, valoró la Universidad de la cual me gradué.

Puedo concluir que me encuentro muy satisfecha de haberme graduado de la UNED, esta es una institución que recomendaría a ojos cerrados y en el futuro me gustaría realizar otros estudios a nivel de posgrado, debido a que este sistema favorece a las personas que con ciertas limitaciones de espacio, tiempo, recursos no podrían obtener una carrera profesional.

\section{Conclusiones}

La educación a distancia es una alternativa viable y eficaz para profesionalizar a los estratos de la sociedad que presentan dificultades con los sistemas tradicionales. Tal y como se ha ilustrado a través de los casos expuestos, la UNED es una institución de educación superior que favorece la inclusión de todos los sectores de la población que quieran y decidan romper barreras, a fin de conseguir un título universitario.

De conformidad con la matricula actual, la UNED ha logrado ubicarse como la segunda universidad más importante en el país. La acreditación de algunas de sus carreras en los últimos años ha demostrado el compromiso de mejora; su innovación constante se evidencia a través de la actualización de los materiales, programas de estudio, capacitación del personal, uso de las TIC, entre otros, todo esto se realiza a fin de formar profesionales capaces de competir en el actual mercado laboral.

Referente a la población compuesta por los privados de libertad, es relevante señalar que la UNED es, en este momento, la única universidad pública costarricense que 
URL: http://www.una.ac.cr/educare

hace posible que individuos que estén en esta condición puedan contar con la posibilidad de cursar una carrera a nivel universitario. De esta forma cumple la universidad con esa responsabilidad social establecida desde su constitución misma, referida a profesionalizar los sectores con mayores limitaciones, los cuales no lograrían sus objetivos en sistemas tradicionales de educación.

No obstante, queda evidenciado que los estudiantes privados de libertad en la UNED tienen limitaciones que retan a la universidad a realizar convenios con el Ministerio de Justicia, a fin de solventar las necesidades detectadas, como la falta de una infraestructura adecuada para atender los estudios, el acceso a los medios virtuales y, en general, a materiales de apoyo que se ofrecen en los cursos, dificultados por las mismas condiciones de control del sistema carcelario que imposibilitan realizar una mediación pedagógica más eficaz.

Es importante destacar que el país ha logrado avanzar en cuanto a la atención de personas con discapacidades, ofreciendo equidad y oportunidades para todos; no obstante, se debe resaltar el hecho de que el sistema de educación a distancia favorece el desarrollo de características especiales en los estudiantes en general y los alumnos con condiciones especiales no quedan excluidos de ellas. Dentro de las más importantes se pueden mencionar: el autocontrol, la disciplina, la capacitad de resolver problemas sin contar con un tutor constantemente, el manejo adecuado del tiempo para lograr objetivos, características que favorecen posteriormente el buen desempeño laboral.

La Sala Constitucional también ha marcado el camino aclarando que si bien las universidades deben adoptar las medidas necesarias y las acciones correspondientes para facilitarles a sus estudiantes el disfrute pleno de las adecuaciones curriculares requeridas (caso de la estudiante no oyente), ello nunca puede ser en perjuicio de la seguridad del resto de los estudiantes (como en el caso del estudiante con problemas psicológicos y conductas impropias), ni tampoco debe ser obligatoria la atención de estudiantes con adecuaciones curriculares significativas, como los casos de retardo mental, ya que ello sí va en contra de los fines de la educación superior de formar profesionales aptos para el ejercicio al servicio de la ciudadanía.

Los desafíos de las universidades a distancia, para ser pertinentes, son el de mantener la certificación de calidad, esto exige a las instituciones la actualización constante; el acceso a las personas con necesidades especiales (de acuerdo con los limites establecidos legal y jurisprudencialmente); la oferta de carreras acordes a las necesidades económicas del país, de tal manera que exista una vinculación entre la demanda laboral y los formadores de mano de obra calificada; el otorgamiento de becas para que personas con dificultades económicas puedan continuar con la educación superior; el acceso a diversos recursos tecnológicos, y el desarrollo de extensión e investigación, que favorezcan la calidad de vida de las personas y de la sociedad en general. 


\section{Referencias}

Arnaiz, P (diciembre, 2000). Las adaptaciones en el currículum universitario. Boletín del Real Patronato, 47, 151-168. Recuperado de http://www.siis.net/documentos/boletin\%20RP/ BRPD47.pdf

Asamblea General de las Naciones Unidas. (2006). Convención de la ONU sobre los derechos de las personas con discapacidad. Recuperado de: http://sid.usal.es/leyes/ discapacidad/10244/3-4-1/convencion-de-la-onu-sobre-los-derechos-de-las-personascon-discapacidad.aspx

Cabero, J. (abril, 2006). Bases pedagógicas del e-learning. Revista de Universidad y Sociedad del Conocimiento 3(1), 1-9.

Castrillo, O M. (junio, 2012). Aprendizaje de los estudiantes universitarios privados de libertad, Centro Penal Calle Real, Liberia. En Fundación CIENTEC (Coord.). Memoria del VIII festival internacional de Matemática. Recuperado de http://www.cientec.or.cr/matematica/2012/ ponenciasVIII/Oscar-Mario-Castrillo.pdf

Código Penal, Sección III, de la libertad condicional, requisitos, artículo (s. f.). Recuperado de http://www.pgr.go.cr/scij/busqueda/normativa/normas/nrm repartidor.asp?param1=N $\underline{\text { RTC\&nValor1 }=1 \& \text { nValor2 }=5027 \& \text { nValor3 }=68813 \& \text { strTipM }=T C}$

Consejo Nacional de Rectores. Oficina de Planificación de la Educación Superior. Comisión de Directores de Planificación. (2011). Plan Nacional de la Educación Superior Universitaria Estatal 2011-2015. Recuperado de http://proyectos.conare.ac.cr/fs/documentos/ Formularios y Documentos/PLAN DE ACCION - PLANES mayo 2011.pdf

Consejo Nacional de Rehabilitación y Educación Especial. (2008). Informe país sobre el cumplimiento de la convención de los derechos de las personas con discapacidad. Recuperado de www2.ohchr.org/SPdocs/CRPD/futuresession/CRPD.C.CRI.1 sp.doc

García, L. (diciembre, 1999). Fundamentos y componentes de la educación a distancia. RIED: Revista Iberoamérica de Educación a Distancia, 2(2), 43-61. Recuperado de http://e-spacio. uned.es/fez/eserv.php?pid=bibliuned:20188\&dsID=fundamentos y componentes.pdf

Organización de Naciones Unidas (ONU). (s. f). Convención sobre los derechos de las personas con discapacidad y protocolo facultativo. Recuperado de http://www.un.org/disabilities/ documents/convention/convoptprot-s.pdf

Programa Estado de la Nación en Desarrollo Humano Sostenible. (2011) Estadísticas de educación. recuperado de http://www.estadonacion.or.cr/index.php/estadisticas/costarica/compendio-estadistico/estadisticas-sociales 
URL: http://www.una.ac.cr/educare

Ramírez, K. (19 de mayo del 2012). Escuela de Ciencias de la Administración de la UNED recibirá certificado de acreditación del SINAES. Acontecer, http://web.uned.ac.cr/acontecer/index. php/a-diario/gestion-universitaria/1388-escuela-de-ciencias-de-la-administracion-de-launed-recibira-certificado-de-acreditacion-del-sinaes.html

Sala Constitucional de la Corte Suprema de Justicia de la República de Costa Rica. (2008a). Resolución 10944-2008 del 4 de julio del 2008. Sistema Costarricense de Información Jurídica. Recuperado de http://200.91.68.20/pj/scij/busqueda/jurisprudencia/jur repartidor.asp?p aram 1=ABC\&param2=1\&param3=FECHA\&param4=DESC\&param5=\&param6=\&cmbDes pacho $=0007 \& \mid$ Resultado $=50$

Sala Constitucional de la Corte Suprema de Justicia de la República de Costa Rica. (2008b). Resolución 11621-2008 de las 10:14 del 25 de julio del año 2008. Sistema Costarricense de Información Jurídica. Recuperado de http://200.91.68.20/pj/scij/busqueda/jurisprudencia/ jur repartidor.asp?param1=TSS\&nValor1=1\&nValor2=419471\&strTipM=T\&strDirSel=dire cto

Sala Constitucional de la Corte Suprema de Justicia de la República de Costa Rica. (2009). Resolución 2773-2009 de las 16:23 del 20 de febrero del 2009. Recuperado de http://200.91.68.20/pj/scij/busqueda/jurisprudencia/jur repartidor.asp?param1=TSS\&nV alor1 $=1 \&$ nValor2=445721\&strTipM=T\&strDirSel=directo

Sala Constitucional de la Corte Suprema de Justicia de la República de Costa Rica. (2010). Resolución No. 10727-2010 de las 10.11 horas del 18 de junio del 2010 (Poder Judicial de la República de Costa Rica). Sistema Costarricense de Información Jurídica. Recuperado de http://200.91.68.20/pj/scij/busqueda/jurisprudencia/jur repartidor.asp?param 1=TSS\&nV alor1=1\&nValor2=488325\&strTipM=T\&strDirSel=directo

Sangrà, A. (mayo, 2002). Educación a distancia, educación presencial y usos de la tecnología: Una tríada para el progreso educativo. Edutec-e. Revista Electrónica de Tecnología Educativa, 15. Recuperado de http://edutec.rediris.es/Revelec2/Revelec15/albert sangra.htm

Scarfó, F. J. (1997). El derecho a la educación en las cárceles como garantía de la educación en derechos humanos. Revista IIDH, 36, pp. 291-324. Recuperado de http://www.corteidh. or.cr/tablas/R06835-11.pdf

Solari, A. y Monge, G. (marzo-abril, 2004). Un desafío hacia el futuro: Educación a distancia nuevas tecnologías y docencia universitaria. Trabajo presentado en el Primer Congreso Latinoamericano de Educación a Distancia. Universidad Nacional de Río Cuarto. República Argentina. Recuperado de http://www.ateneonline.net/datos/96 03 Birri Roberto.pdf

Universidad Estatal a Distancia (UNED). (1977). Ley de creación de la Universidad Estatal a Distancia (UNED). Recuperada de http://www.uned.ac.cr/Reglamento/documents/ LEYDECREACION.pdf 
Universidad Estatal a Distancia (UNED). (2009). Estatuto Orgánico. San José, Cota Rica: Centro de información, documentación y recursos bibliográficos (Área de información y documentación institucional). Recuperado de http://estatico.uned.ac.cr/cidi/reglamento/ documents/ESTATUTOORGANICOCIDREB20090CT.pdf

\section{Cómo citar este artículo, según APA:}

Ulate, I. y Vargas. E. (2012). La educación a distancia en la profesionalización de las personas con discapacidad y privados de libertad. Revista Electrónica Educare, 16(3), 157-179. Consultado de http://www.revistas.una.ac.cr/index.php/EDUCARE/issue/current

Nota: Para citar este artículo en otros sistemas puede consultar el hipervínculo "Como citar el artículo" en la barra derecha de nuestro sitio web:

http://www.revistas.una.ac.cr/index.php/EDUCARE/index 